\title{
The Research on Color Grading of Green Jade Images Based on HSL Chromaticity Analysis
}

\author{
Lili Zhang ${ }^{\text {a }}$ and Xinqiang Yuan \\ Gemmological Institute, China University of Geosciences, Wuhan, 430074, China
}

\begin{abstract}
This study aims to establish a simple and convenient method suitable for the market to grade the jade colors. Based on the HSL color model and with the assistance of the HSL analysis on green jade images acquired by camera flashlights, this study obtains the following six kinds of jade colors: melon green, emerald green, yellow green, bean green, light bean green, and light green, based on which the law governing the values and the value range can be determined, and the respective HSL value ranges ,together with their threshold values, for the seven green jades can be finally found, thus laying an important foundation for further researches on computer-based quick grading. It is also hoped that this method will be widely applied and promoted in the marketplace and realizes our ultimate goal of setting guidelines to commercial jade prices.
\end{abstract}

Keywords: Green jade; color evaluation; image analysis method; HSL value

\section{Introduction}

Image analysis method, also known as digital color camera colorimetric analysis method, will be applied in this research. Digital color cameras are equipped with CCD color chips full of lightsensitive photosensitive diodes that contain the so-called pixels. Each pixel in the CCD color chip is individually covered with red, green and blue filters so as to only sense the light in the corresponding color. For instance, a jade imaged to the CCD color chip through a digital camera's lens will be sensed by the pixel of the chip, after which electrical signals will be formed. The strength of the electrical signals will be expressed within the range of 0-255 and saved to the memorizer. These signals can be read on a computer, and the color images displayed on the screen. If chromaticity analysis is adopted for reading the signals, quantitative data describing the image colors, such as the R, G and B data, can be obtained. In spite of the fact that RGB data (also referred to as tristimulus values) are the most basic colorimetric parameters, namely, any color can be reproduced via the mixture of the three colors red (R), green (G) and blue (B), and they stand for the quantity of the RGB contained in these colors, such a color description differs from the color description practices of the author and is inconvenient to use. Therefore, RGB values are converted to the more widely-used values of hue (H), saturation (S), and lightness (L).

\section{HSL Color Model}

\footnotetext{
${ }^{a}$ Corresponding author : lilili29@sina.com
} 
This research focusing on acquiring the color image of ordinary green jade on the market has its basis on the HSL color model, a model catering to human senses of colors. This model, as a color system established by the scholar Smith, is subject to artists' visual sense on color and lustre, light and shade and hue[2]. In the HSL model, all colors are described with the three basic parameters: hue, saturation and lightness. Therefore, users without knowledge of color mixture can specify colors as well. The difference between the HSL and the common RGB color models lies in that the latter is a psychophysical color system, while the former perception one[3].

\section{HSL Colorimetric Test for Green Jade}

\subsection{Introduction of HSL Colorimetric Test for Green Jade}

To better understand the colorimetric characteristics of green jade, the author has acquired a sample of 1590 green jade photos taken by cameras with flashlights, and selected 70 effective photos among the sample for this research. Next, obtain the HSL chromatic value for each photo with the help of relevant software by setting to the appropriate value on the HSL color-block coordinate axis, as shown in Figure $1(\mathrm{H}=120)$.

Though green jade exemplifies a wide range of color changes, a few such change series can still be observed and distinguished via naked eyes. The experiments divided the jades into various types, and a unanimous result of 6 distinctive color types is obtained. According to the color depth sequence from visual sense, the colors well-recognized in the jade industry are: melon green, emerald green, yellow green, bean green, light bean green, light green and pale green. In this experiment, HSL test was carried out on the 6 colors[4][5].

In addition, since jade has a complex color distribution, with some evenly distributed, while others are not, a color analysis solely dependent on the HSL values will with no doubt lead to bias. Therefore, for a more objective result, the author has in particular applied the software to get the average value of the jade image colors and selected the single point values for representative colors on the jade image. Besides, coordinate points indicating different colors for each kind of jade are used to mark on the color block coordinates (see Figure 1). Specific HSL analysis data are shown in Table 1 below, where data in the first row under the figure represent the average green values. For convenience, data recording is simplified, only data are kept here with their units omitted. For instance, in the table the first photo' HSL values are $128^{\circ}, 25 \%$ and $52 \%$, which are simplified as 128,25 and 52 .

\subsection{Test data results as well as the distribution of the corresponding color block coordinate chart}

Table 1. Images acquired and HSL parameters of green jade

\begin{tabular}{|c|c|c|c|c|c|c|c|}
\hline \multirow{5}{*}{$\begin{array}{c}\text { Melon } \\
\text { green }\end{array}$} & 1 & 2 & 3 & 4 & 5 & 6 & 7 \\
\hline & $128,25,52$ & $135,28,47$ & $124,32,41$ & $131,55,25$ & $128,31,48$ & $126,43,47$ & $141,30,18$ \\
\hline & 8 & 9 & 10 & 11 & & & \\
\hline & & & & & & & \\
\hline & & & & & & & \\
\hline
\end{tabular}




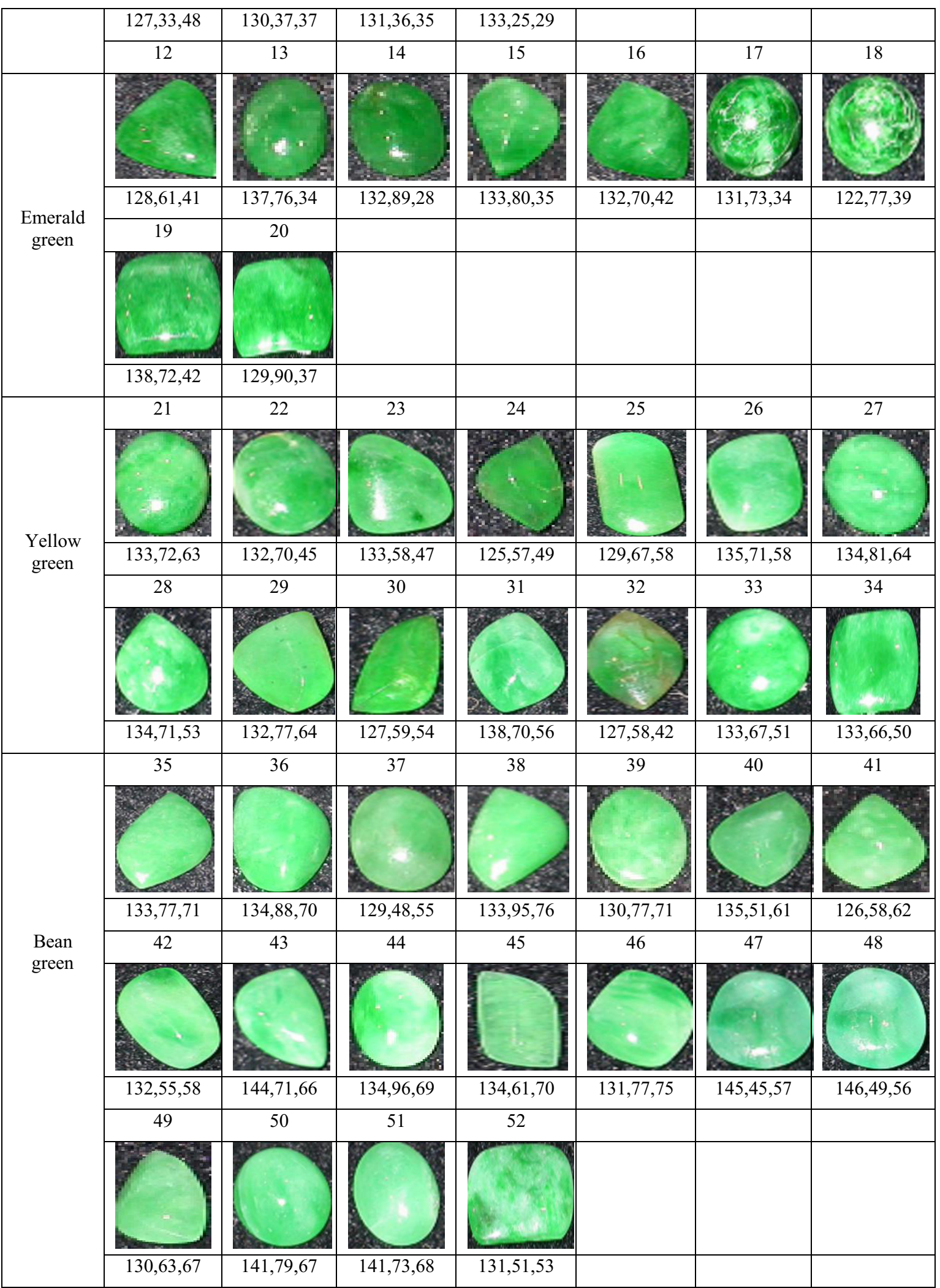




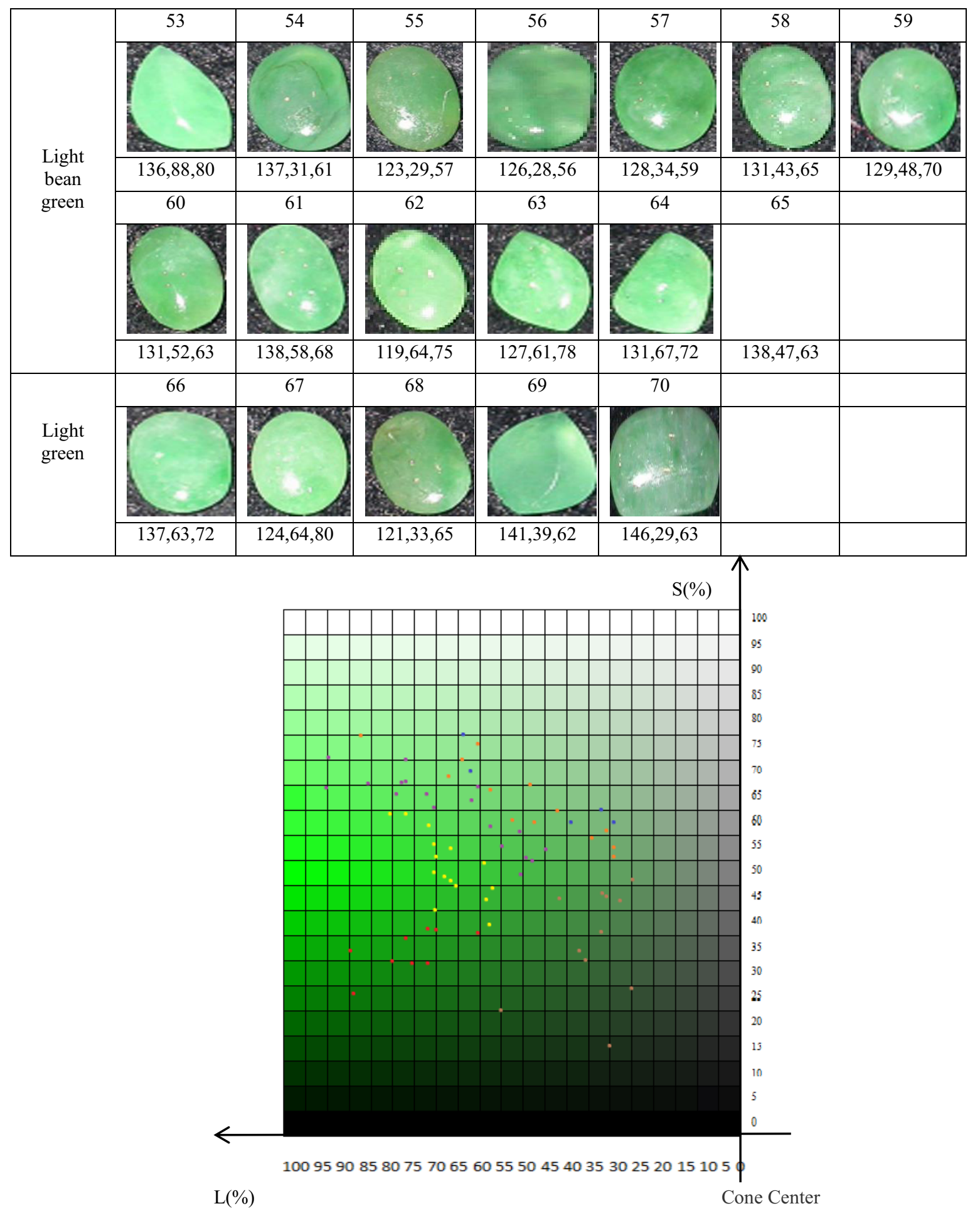

*S (saturation )*L (lightness)

*Melon green is marked with brown points, emerald green is marked with bright-red points, yellow green is marked with yellow points, bean green is marked with purple points, light bean green is marked with reddish orange points, light green is marked with blue points and pale green is marked with white points.

Figure 1. HSL color analysis color block coordinate of green jade-- average value 


\section{Test analysis and discussion}

The above HSL value test result shows that: The 6 colors of green jade are different, and based on such difference, various colors can be differentiated. HSL values of each color have their own scope of law, which can be seen obviously from the distribution characteristics analysis of the HSL values of green jade in the color block coordinates shown above. Jades with bright color and uniform color are characterized in high saturation of above $80 \%$; moderate lightness, mostly of 20-30; hue of 120-149; which are green. Those whose hue is above 150 will have blue tone, affecting the green beautiful degree of the jade. Those whose lightness is below 10 are too dark, which can be classified into black jadeite. Those whose lightness is above 80, and saturation is below 20 can be classified into white jade due to light color, which is close to white (or colorless). The green beautiful degree of jade is mainly affected by saturation and lightness, definite relevance lies between the color saturation and lightness, while hue is secondary.

Problems emerged during the test: Since jade color is featured by hierarchy and complexity, the most common jades in the market often do not have the relatively single color change like diamond, lack of progressive color gradation. Therefore, even if differentiated with naked eyes, under the circumstance where samples are uniform, such jade with close color grade will have inconsistent color grading. The authors find from this test that, when analyzing the HSL values, for green jades with close color grade, boundary overlap or confusion of the color values will occur.

\section{CONCLUSIONS}

Through the above test and analysis of the HSL values of the green jade, HSL values of the seven kinds of green jade can be obtained, and through the analysis of these values, the seven kinds of color of the green jade can be numeralized. Besides, numerical range and threshold interval of each color of jade can be pinpointed (see table 2 ). This research makes the fast digitization of jade color possible, which can be served as the colorimetric indicators for color grading of green jade, thus, creating a new way for the fast and effective grading of jade color. Namely, color grade of the jade can be obtained quickly and precisely by image analysis of the HSL values of each jade. Meanwhile, it has laid an important foundation for the late stage research of computer jade color automatic grading system.

Table 2. HSL Parameter Distribution Scope of All Kinds of Colors of Green Jade

\begin{tabular}{|c|l|c|c|c|}
\hline Color grade & Color name & Hue (H) & Saturation (S) & Lightness (L) \\
\hline 1 & Melon green & $120-150$ & $20-50$ & $15-40$ \\
\hline 2 & Emerald green & $120-135$ & $60-90$ & $28-40$ \\
\hline 3 & Yellow green & $125-138$ & $60-80$ & $45-60$ \\
\hline 4 & Bean green & $130-140$ & $50-80$ & $60-70$ \\
\hline 5 & Light bean green & $125-135$ & $20-60$ & $50-70$ \\
\hline 6 & Light green & $120-140$ & $20-60$ & $60-80$ \\
\hline
\end{tabular}




\section{References}

1. Mo Site, Liu Tianqi, Li Bixiong., HSL Color Space-Based Automatic White Balance Algorithm. Journal of Sichuan University (Engineering Science Edition), 6 (45): 95-99(2013)

2. Liu Xiao, Jiang Gnagyi, Wu Xunwei., HSL Space-based Color Morphological Transformation. Journal of Circuits and Systems, 3 (7): 52-56(2002)

3. Hen Zhang: Optimization of Green Jade color grading standard (MS., Kunming University of Science and Technology, China,14-24(2010)

4. Yuan Xinqiang., Application Jade Gemology. Wuhan: China University of Geosciences Press Co., Ltd.:91(2009)

5. Hui Zhang, Beili Zhang, Manjun Wang: Application of Method of Colour Measurement in Colour Appraisement of Jadeite Jade, Journal of Gems and Gemmology, Vol.8 No.3:16-20(2006) 